\title{
Anomalous Doppler effects in phononic band gaps
}

\author{
Xinhua $\mathrm{Hu},{ }^{1, *}$ Zhihong Hang, ${ }^{1}$ Jensen $\mathrm{Li}^{1}{ }^{1} \mathrm{Jian} \mathrm{Zi},{ }^{2}$ and C. T. Chan ${ }^{1}$ \\ ${ }^{1}$ Department of Physics, Hong Kong University of Science and Technology, Clear Water Bay, Kowloon, Hong Kong, China \\ ${ }^{2}$ Surface Physics Laboratory (National Key Lab), Fudan University, Shanghai 200433, People's Republic of China
}

(Received 19 July 2005; published 30 January 2006)

\begin{abstract}
Doppler effects in periodic acoustic media were studied theoretically and experimentally. Analytical formulas are derived using the Green's function formalism. We found that a far field observer cannot hear the sound inside a band gap from a stationary source, but a moving source can be heard even if the frequency is inside the gap, and the Doppler shifts can be inverted or anomalously large.
\end{abstract}

DOI: 10.1103/PhysRevE.73.015602

PACS number(s): 43.40.+s, 63.20.-e, 42.70.Qs

The propagation of classical waves in inhomogeneous periodic media, including electromagnetic waves [1], elastic and sonic waves [2], and liquid surface waves [3], has received considerable attention [4-11]. The effect of Bragg scattering leads to forbidden frequency ranges called band gaps, and the strongly modified dispersion relations and density of states (DOS) lead to many intriguing phenomena such as the inhibited spontaneous emission, localization of light [1], the ultrafast tunneling [4], superlensing effect [5], anomalous Cerenkov radiation [6], and giant Lamb shift [7].

To date, most of the studies have dealt with a stationary source and the emission of a moving source in the band-gap media has not been studied. In free space, an observer will detect a higher frequency from a moving source when the source and observer approach each other. The Doppler shifts can be reversed in some dispersive media [12]. Inverse Doppler effects in the pass bands have also been demonstrated using a moving boundary in electromagnetic band-gap media such as photonic crystals [8] and transmission lines [9]. However, the Doppler effects for a moving source emitting signals with frequencies inside band gaps in which the media carries no propagating modes have not been investigated.

In this paper, we considered a moving sound source in a quasi-one-dimensional (quasi-1D) phononic crystal (an acoustic periodic waveguide) [10] and we focused on the Doppler effect with the source frequency lying inside a band gap. The Green's function formalism was first applied to obtain general formulas for Doppler effects in the phononic crystal. Interesting asymmetry between the effects of motion of source and observer in phononic structures were predicted and verified experimentally: while sound from a stationary source at a frequency inside the gap cannot be heard by a distant observer (even if it moves), sound from a moving source can be heard at different frequencies. These Doppler frequency shifts can be normal, inverse, and can be large (triple the normal value), leading to anomalous Doppler effects in phononic band gaps.

Consider a rigid, straight tube, circular in cross section, with periodic diameter variations described by $D(x+a)$ $=D(x)$, where $a$ is the lattice constant (see Fig. 1). When its

\footnotetext{
*Present address: Ames Lab and Department of Physics and Astronomy, Iowa State University, Ames, Iowa 50011, USA.
}

wavelength $\lambda>2 D$, any sound field in the tube will depend only on $x$ and obey a one-dimensional (1D) wave equation $[10,13,14]$

$$
\frac{\partial}{\partial x}\left(\frac{S(x)}{\rho} \frac{\partial}{\partial x} P(x, t)\right)-\frac{S(x)}{\rho v_{a}^{2}} \frac{\partial^{2}}{\partial t^{2}} P(x, t)=\frac{\partial}{\partial t} J(x, t),
$$

where $S=\pi D^{2} / 4$ is the cross-sectional area of tube, $P$ is the pressure, $J$ is the sound source, $\rho$ is the mass density of air, and $v_{a}$ is the sound speed in air. In the following, this periodically structured tube will be referred to as a PS tube, and a nonstructured tube with constant cross section will be termed an NS tube.

When $J(x, t)=0$, the eigenmode can be written as

$$
P(x, t)=u_{\kappa, \omega}(x) e^{i(\kappa x-\omega t)},
$$

where $u_{\kappa, \omega}(x)=u_{\kappa, \omega}(x+a)$ is a periodic function, $\omega$ is the angular frequency, and $\kappa$ (when $\kappa$ is real) is the Bloch wave vector. For a two-diameter unit cell $\left[S(x)=S_{1}\right.$ when $-d_{1}<x<0, S(x)=S_{2}$ when $\left.0<x<d_{2}, d_{1}+d_{2}=a\right]$, the disper-
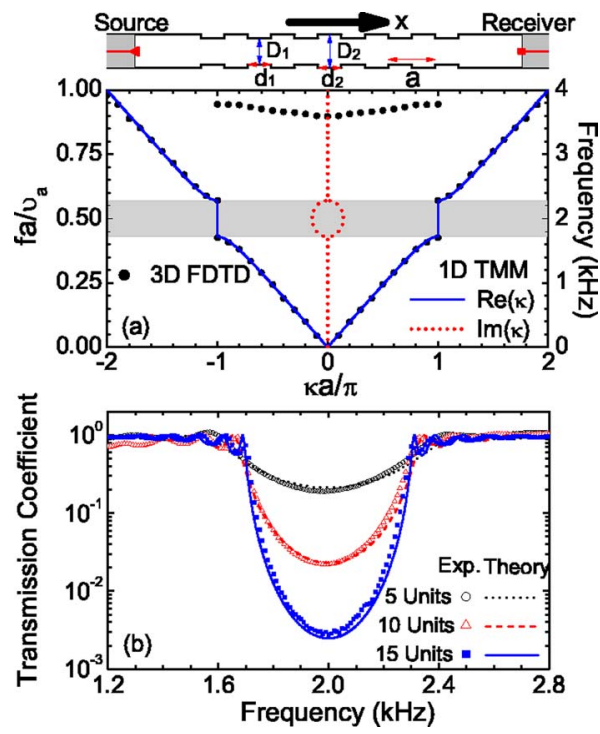

FIG. 1. (Color online) (a) Acoustic band structures and (b) transmission spectrum for a circular tube with the periodic structure $D_{1}=4.8 \mathrm{~cm}, D_{2}=6.0 \mathrm{~cm}, d_{1}=d_{2}=4.25 \mathrm{~cm}, a=8.5 \mathrm{~cm}$. The inset shows the geometry of the tube. 
sion relationship can be obtained by a $2 \times 2$ transfer matrix method (TMM) [10,15],

$$
\cos (\kappa a)=\cos \left(\frac{\omega a}{v_{a}}\right)-\frac{1}{2}\left(\frac{S_{2}}{S_{1}}+\frac{S_{1}}{S_{2}}-2\right) \sin \left(\frac{\omega d_{1}}{v_{a}}\right) \sin \left(\frac{\omega d_{2}}{v_{a}}\right) .
$$

For a given $\omega$, two solutions of $\pm \kappa[\operatorname{Re}(\kappa), \operatorname{Im}(\kappa)>0]$ exist and $u_{-\kappa, \omega}(x)=u_{\kappa, \omega}(x) \equiv u_{\omega}(x)$ [16]. For an NS tube, the eigenmodes will be plane waves with $|\kappa|=\omega / v_{a}$, and $u_{\kappa, \omega}(x)=1$.

For a stationary, harmonic, plane sound source $J(x, t)$ $=\delta\left(x-x^{\prime}\right) e^{-i \omega t}$, where $\omega$ and $x^{\prime}$ designate the angular frequency and position of the source, the solution will be $P^{ \pm}(x, t)=B^{ \pm} u_{\omega}(x) \exp \left( \pm i \kappa_{\omega} x-i \omega t\right)$ where + and - denotes $x>x^{\prime}$ and $x<x^{\prime}$, respectively [16]. Using $P^{+}\left(x^{\prime}, t\right)$ $=P^{-}\left(x^{\prime}, t\right)$ and $\left[S\left(x^{\prime}\right) / \rho\right]\left((\partial / \partial x) P^{+}\left(x^{\prime}, t\right)-(\partial / \partial x) P^{-}\left(x^{\prime}, t\right)\right)$ $=-i \omega$ [obtained by integrating Eq. (1) with $\int_{x^{\prime}-\sigma}^{x^{\prime}+\sigma} d x$ and $\sigma \rightarrow 0$ ], the solution $P(x, t)$ for $J(x, t)=\delta\left(x-x^{\prime}\right) e^{-i \omega t}$ can be obtained,

$$
Q_{\omega}\left(x, x^{\prime}, t\right)=\frac{-\rho \omega u_{\omega}(x)}{2 \kappa_{\omega} S\left(x^{\prime}\right) u_{\omega}\left(x^{\prime}\right)} e^{i \kappa_{\omega}\left|x-x^{\prime}\right|-i \omega t} .
$$

In addition, the general solution of Eq. (1) with an arbitrary sound source $J(x, t)$ can be written as the integral $\int_{-\infty}^{+\infty} d x^{\prime} \int_{-\infty}^{+\infty} d t^{\prime} J\left(x^{\prime}, t^{\prime}\right) \int_{-\infty}^{+\infty} d \omega Q_{\omega}\left(x, x^{\prime}, t-t^{\prime}\right)$.

Consider, then, two cases: the first (I) with a stationary source $J(x, t)=\delta\left(x-x_{s}\right) e^{-i \omega_{0} t}$ and a moving receiver at position $x=v_{r} t$ ( $v_{r}$ is the velocity of the receiver), and (II) a moving source $J(x, t)=\delta\left(x-v_{s} t\right) e^{-i \omega_{0} t}\left(v_{s}\right.$ is the velocity of source) and a stationary receiver located at $x_{r} . \omega_{0}$ is the original angular frequency of the source, and $v_{s}\left(v_{r}\right)>0$ indicates that the source (receiver) is moving towards $+\infty$.

Case I: the signal received will be $Q_{\omega_{0}}\left(v_{r} t, x_{s}, t\right)$ $=\Sigma_{G}\left[-\rho \omega_{0} A_{\omega_{0}, G} / 2 \kappa_{\omega_{0}} S\left(x_{s}\right) u_{\omega_{0}}\left(x_{s}\right)\right] e^{ \pm i \kappa_{\omega_{0}} x_{s}-i \omega t}$, where

$$
\omega=\omega_{0}+\left(G \pm \kappa_{\omega_{0}}\right) v_{r}
$$

$u_{\omega}(x)=\Sigma_{G} A_{\omega, G} e^{-i G x}(G=2 m \pi / a, m=0, \pm 1, \pm 2, \ldots)$, and + $(-)$ occurs when the receiver is moving towards (away from) the source. For a NS tube $\left(A_{\omega, G}=\delta_{G, 0}\right)$, Eq. (5) reduces to the common Doppler formula in free space: $\omega=\omega_{0}\left(1 \pm v_{r} / v_{a}\right)$.

Case II: the received signal will be

$$
\begin{aligned}
\int_{-\infty}^{+\infty} d x^{\prime} \int_{-\infty}^{+\infty} d t^{\prime} \delta\left(x^{\prime}-v_{s} t^{\prime}\right) e^{-i \omega_{0} t^{\prime}} \int_{-\infty}^{+\infty} d \omega Q_{\omega}\left(x_{r}, x^{\prime}, t-t^{\prime}\right) \\
=\sum_{G} \int_{-\infty}^{+\infty} d \omega F_{G}(\omega)\left[\rho \omega u_{\omega}\left(x_{r}\right) / 2 \kappa_{\omega}\right] \\
\quad \times e^{i}\left[\left(\omega-\omega_{0} / v_{s}\right)-G\right] x_{r}-i \omega t
\end{aligned}
$$

where $\quad F_{G}(\omega)=A_{\omega, G}^{\prime}\left\{1 /\left[\omega-\left[\omega_{0}+\left(G+\kappa_{\omega}\right) v_{s}\right]\right]\right.$ $\left.-1 /\left[\omega-\left[\omega_{0}+\left(G-\kappa_{\omega}\right) v_{s}\right]\right]\right\}$ and $1 / S(x) u_{\omega}(x)=\Sigma_{G} A_{\omega, G}^{\prime} e^{-i G x}$ [17]. $F_{G}(\omega)$ has peaks at

$$
\omega=\omega_{0}+\left(G \pm \kappa_{\omega}\right) v_{s},
$$

where $+(-)$ occurs when the source is moving towards (away from) the receiver. When $\omega$ is near the first band gap
$(\operatorname{Re}(\kappa) \approx \pi / a)$, the Doppler shift in the frequency (real part) will be

$$
\Delta f \equiv f-f_{0} \approx\left( \pm \frac{1}{2}+m\right) \frac{v_{s}}{a} .
$$

For a NS tube $\left[A_{\omega, G}^{\prime}=(1 / S) \delta_{G, 0}\right]$, Eq. (6) reduces to the common Doppler formula in free space: $\omega=\omega_{0} /\left(1 \mp v_{s} / v_{a}\right)$ and $\Delta f \approx \pm f_{0} v_{s} / v_{a}$ for $v_{s} \ll v_{a}$.

In free space, a single up-shifted (down-shifted) Doppler peak can be detected when the source is moving towards (away from) the receiver. However, the Doppler effect in a PS tube can be very different. In case I (case II), the receiver can detect many Doppler peaks with angular frequencies given by Eq. (5) [Eq. (6)] and intensities proportional to $A_{\omega_{0}, G}\left(A_{\omega, G}^{\prime}\right)$. For Eq. (5) corresponding to a moving observer, when $\omega_{0}$ is inside the band gap, $\kappa_{\omega_{0}}$ is complex, and thus the Doppler peak will have an apparent complex frequency according to Eq. (5) which can be interpreted as a finite lifetime $\tau \sim 1 / \operatorname{Im}\left(\kappa_{\omega_{0}}\right) v_{r}$ of the Doppler peak. The source does not "die," but the receiver hears a decaying signal because the sound is transmitted via evanescent waves through increasing distance and the exponential decay is governed by the imaginary component of $\kappa_{\omega_{0}}$. We note that Eq. (6) is different from (5) as the $\kappa_{\omega}$ corresponds to $\omega$ and not $\omega_{0}$ as in (5), and thus allows for a distant observer to hear the sound with $\omega$ outside the band gap even when $\omega_{0}$ is inside the gap.

So for a distant sound source with a frequency $\omega_{0}$ in the band gap, a very intriguing phenomenon can be predicted: the observer (whether stationary or moving) cannot hear any sound in case I. But as soon as the source begins to move, the sound can be heard at different frequencies (case II). The frequency shift can be inverse, or much larger than the common Doppler effect.

To demonstrate the anomalous Doppler effect predicted above, a polyvinyl chloride (PVC) tube (wall thickness $=5 \mathrm{~mm}$ ) with a periodic structure was fabricated such that $a=8.5 \mathrm{~cm}, d_{1}=d_{2}=4.25 \mathrm{~cm}, D_{1}=4.8 \mathrm{~cm}$, and $D_{2}=6.0 \mathrm{~cm}$. Figure 1(a) shows the acoustic band structures for this tube calculated by the 1D TMM [Eq. (3)] and a fully fledged three-dimensional (3D) finite-difference time-domain (FDTD) method [18]. For frequencies below $3.59 \mathrm{kHz}$ (above which transverse modes occur), the 1D theory gave almost the same results as the full 3D simulations. A band gap was found extending from 1.72 to $2.28 \mathrm{kHz}$, in which $\kappa$ had an imaginary part and sound waves could not propagate.

Transmission measurements were performed by connecting this PS tube (and an NS tube for comparison) with two tubes which contained a small sound source and a receiver (cross-sectional area $\approx 0.05 S_{2}$ and $0.02 S_{2}$ ) and were plugged with sound-absorbing cotton (absorption $>99 \%$ for $1 \sim 3 \mathrm{kHz}$ ) at the free ends (see Fig. 1) [11]. Single frequency excitation and phase lock-in techniques were used to improve the signal-to-noise ratio [11]. The measured amplitude transmission coefficients $T$ are plotted in Fig. 1(b) and compared with the theoretical results from the 1D TMM calculations. Good agreement is apparent. Between 1.72 and $2.28 \mathrm{kHz}$, transmission was very small, and the dip in trans- 


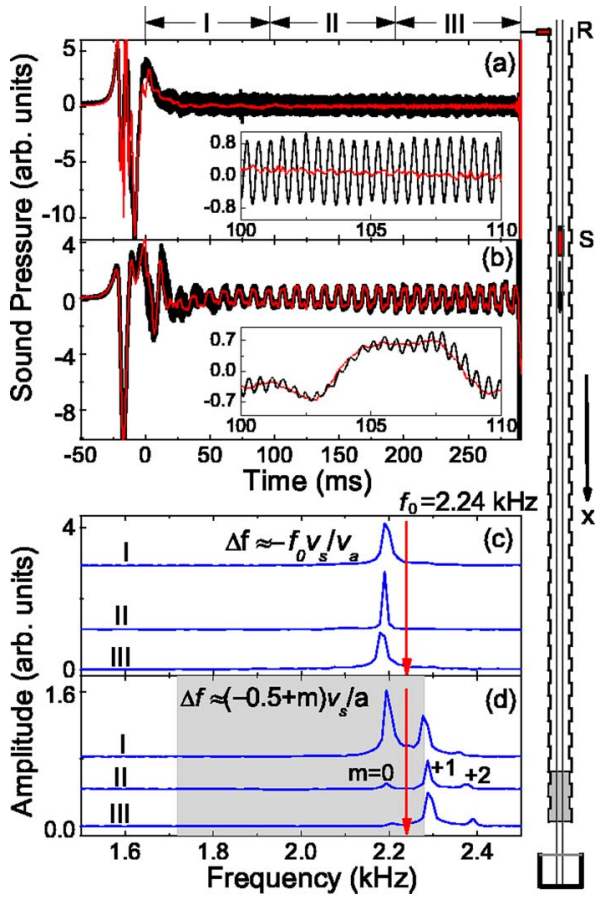

FIG. 2. (Color online) Measured signals (black line) and noise (red line) for a moving sound source (a) in a $2.6 \mathrm{~m}$ long NS tube, and (b) in a PS tube with 30 unit cells. (c) and (d) show the frequency spectra of the signals in (a) and (b), respectively. The PS tube had the same parameters as that in Fig. 1. The insets in panels (a) and (b) are expanded views from 100 to $110 \mathrm{~ms}$.

mission was deeper for the longer tube $\left[T_{\omega}(L) \approx e^{-\operatorname{Im}\left(\kappa_{\omega}\right) L}\right.$ was found, where $L$ is the length of the PS tube]. This agrees well with the gap shown in the theoretical band structures.

The Doppler effect on a moving sound source was then measured in a vertical tube (see Fig. 2), where gravity was used to produce the source velocity. A microphone was fixed at the top of the tube and the sound absorbing cotton was inserted on the bottom. To protect the source, an elastic shock absorber was positioned under the tube. Then a small sound source with single frequency $f_{0}=2.240 \mathrm{kHz}$ [19] was dropped from a position $5.2 \mathrm{~m}$ above the bottom end of the tube, to fall vertically along two taut nylon threads. The sound signals and noise (recorded by turning on and off the source, sampling rate $=48 \mathrm{kHz}$ ) [19] are shown in Fig. 2(a) for a 2.6-m-long NS tube $(D=5.0 \mathrm{~cm})$, and in Fig. 2(b) for a PS tube with 30 unit cells. From about $t=0 \mathrm{~ms}$, the signal becomes strong, showing that sound from the source begins to enter the tube. At $t=291 \mathrm{~ms}$, a shock signal occurred due to the attachment of source with the cotton, indicating the leaving of source from the tube.

Fast Fourier transforms (FFTs) were then applied to the sound signals in three time sections: I (0 to $97 \mathrm{~ms}$ ), II (98 to $196 \mathrm{~ms}$ ), and III (197 to $291 \mathrm{~ms}$ ). It is found that noise from the moving source was mainly in the low frequency range $(<300 \mathrm{~Hz})$ and had very small amplitudes in the frequency range of interest ( 1 to $3 \mathrm{kHz}$ ) compared with the signals. In the PS tube, the moving body generated a periodic airflow causing noise with a fundamental frequency $f_{s}=v_{s} / a$. For the noise in Fig. 2(b), $f_{s}$ was found to be 83 (I),

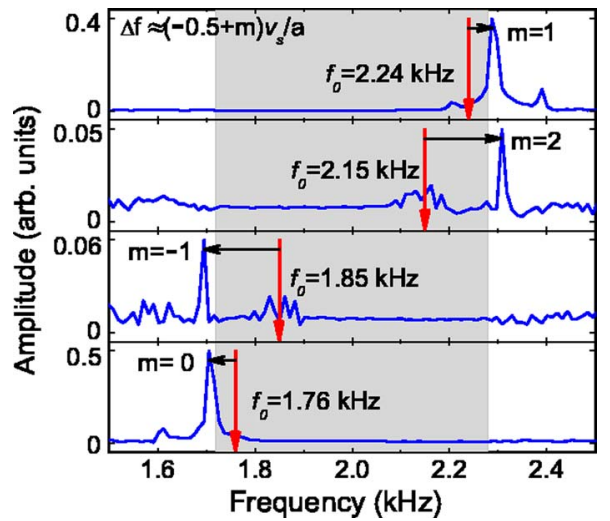

FIG. 3. (Color online) Frequency spectrum [similar to Fig. 2(d) III] of the signals from a distant moving sound source with varying $f_{0}$ in a PS tube. The PS tube had the parameters listed in Fig. 1. The intensity is normalized to the intensity of a NS tube.

93 (II), and 103 (III) Hertz (accuracy $= \pm 5 \mathrm{~Hz}$ ). So the average velocities of the source $v_{s}=7.1$ (I), 7.9 (II), and 8.8 (III) $\mathrm{m} \mathrm{s}^{-1}$ could be obtained.

Figures 2(c) and 2(d) show the frequency spectra for the signals in Figs. 2(a) and 2(b), respectively. A steady normal Doppler peak was found at $f=2.190 \mathrm{kHz}$ in Fig. 2(c), where the Doppler shift $\Delta f=-50 \mathrm{~Hz}$ (Sec. II) agrees well with the theoretical value $\Delta f \approx-f_{0} v_{s} / v_{a}=-52 \mathrm{~Hz}$. However, three Doppler peaks with $\Delta f=-46$ (normal), +47 (inverse), $+136 \mathrm{~Hz}$ (large inverse) were found, as shown in Fig. 2(d) (Sec. II). This agrees well with the theoretical values $\Delta f \approx\left(-\frac{1}{2}+m\right)_{v_{s}} / a=-47 \mathrm{~Hz}$ for $(m=0),+47 \mathrm{~Hz}$ for $(m=1)$, $+140 \mathrm{~Hz}$ for $(m=2)$. The normal peak was found to have a high intensity only when the source was near the receiver. But when the source was far from the receiver, this normal peak became very weak and eventually only the inverse peak dominates the spectra.

Doppler measurements were performed in the PS tube with varying frequencies of the source $f_{0}$ in the band gap. The frequency spectrum when the source was far from the receiver (Sec. III) is shown in Fig. 3. When $f_{0}=2.15 \mathrm{kHz}$, a large inverse Doppler shift $\Delta f=+159 \mathrm{~Hz}$ was observed, relating to the theoretical $m=2$ peak with $\Delta f=+155 \mathrm{~Hz}$. When

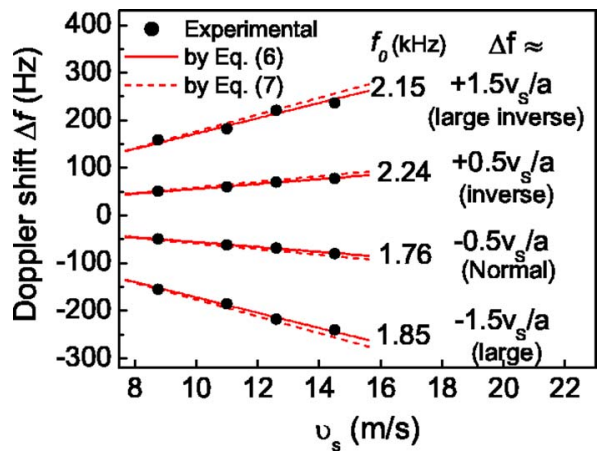

FIG. 4. (Color online) Doppler shifts for $f_{0}$ inside the band gap as a function of the velocity of the source for a distant moving sound source in a PS tube [similar to Fig. 2(d) III]. The PS tube was as described in Fig. 1. 
$f_{0}$ went deeper into the gap, only higher-order Doppler peaks could break out. But they had much weaker intensities and could not be distinguished from the noise. When $f_{0}$ was decreased further, Doppler peaks could be heard again, but with normal (negative) shift signs. When $f_{0}=1.85 \mathrm{kHz}$, a large $\Delta f=-155 \mathrm{~Hz}$ was observed, corresponding to the $m=-1$ peak (theoretical $\Delta f=-155 \mathrm{~Hz}$ ). When $f_{0}=1.76 \mathrm{kHz}$, the $m=0$ peak with a normal Doppler shift $\Delta f=-51 \mathrm{~Hz}$ (calculated $\Delta f=-52 \mathrm{~Hz}$ ) could be detected.

To further verify the relation between $\Delta f$ and $v_{s}$, Doppler measurements were performed varying the source velocities $v_{s}$. The results are shown in Fig. 4. Four kinds of Doppler effects were observed when $f_{0}$ was in the band gap:
$\Delta f \approx-0.5 v_{s} / a$ at $1.76 \mathrm{kHz}$ (normal), $\Delta f \approx-1.5 v_{s} / a$ at $1.85 \mathrm{kHz}$ (large), $\Delta f \approx 1.5 v_{s} / a$ at $2.15 \mathrm{kHz}$ (large inverse), $\Delta f \approx 0.5 v_{s} / a$ at $2.24 \mathrm{kHz}$ (inverse).

Anomalous acoustic Doppler effects were thus demonstrated experimentally in a periodically structured tube, and the observations agreed well with theoretical predictions. Inverse and large Doppler shifts are found for a distant moving source with frequencies in the band gap.

The authors thank X. X. Zhang, O. K. C. Tsui, L. Zhou, and H. Q. Li, for interesting discussions. This work was supported by Hong Kong's Research Grant Council through Grant No. 600305.
[1] E. Yablonovitch, Phys. Rev. Lett. 58, 2059 (1987); S. John, ibid. 58, 2486 (1987).

[2] E. N. Economou and M. M. Sigalas, Phys. Rev. B 48, 13434 (1993); M. S. Kushwaha et al., Phys. Rev. Lett. 71, 2022 (1993); J. V. Sanchez-Perez et al., Nature (London) 378, 241 (1995).

[3] T. Chou, Phys. Rev. Lett. 79, 4802 (1997); M. Torres et al., Nature (London) 398, 114 (1999).

[4] C. Spielmann et al., Phys. Rev. Lett. 73, 2308 (1994); S. Yang et al., ibid. 88, 104301 (2002).

[5] M. Notomi, Phys. Rev. B 62, 10696 (2000); S. Yang et al., Phys. Rev. Lett. 93, 024301 (2004); X. Hu et al., Phys. Rev. E 69, 030201(R) (2004).

[6] C. Luo et al., Science 299, 368 (2003).

[7] X. H. Wang et al., Phys. Rev. Lett. 93, 073901 (2004).

[8] E. J. Reed, M. Soljacic, and J. D. Joannopoulos, Phys. Rev. Lett. 90, 203904 (2003); 91, 133901 (2003).

[9] N. Seddon and T. Bearpark, Science 302, 1537 (2003); A. M. Belyantsev and A. B. Kozyrev, Tech. Phys. 45, 747 (2000); 47, 1477 (2002); A. B. Kozyrev and D. W. van der Weide, Phys. Rev. Lett. 94, 203902 (2005).

[10] C. E. Bradley, J. Acoust. Soc. Am. 96, 1844 (1994); J. N. Munday et al., ibid. 112, 1353 (2002).

[11] Z. Liu et al., Science 289, 1734 (2000); K. M. Ho et al., Appl. Phys. Lett. 83, 5566 (2003).

[12] V. G. Veslago, Sov. Phys. Usp. 10, 509 (1968).

[13] In this case, the volume velocity $U$ and the pressure $P$ should be continous. So the $3 \mathrm{D}$ acoustic wave equations become as $(\partial / \partial t) P(x, t)=-\left[\rho_{a} v_{c}^{2} / S(x)\right]((\partial / \partial x) U(x, t)+J(x, t))$, $(\partial / \partial t) U(x, t)=-\left[S(x) / \rho_{a}\right](\partial / \partial x) P(x, t)$ and Eq. (1) can be obtained. These equations are also valid for 1D photonic crystals if $P, U, S / \rho_{a} v_{c}^{2}, \rho_{a} / S$, and $J$ is replaced by the electric field $E_{y}$, the magnetic field $H_{z}$, permitivity $\varepsilon$, permeability $\mu$, and electric current $J$, respectively.

[14] P. M. Morse, Vibration and Sound (McGraw-Hill, New York, 1948); F. Fahy, Foundations of Engineering Acoustics (Academic Press, San Diego, 2001).

[15] See, e. g., K. Busch, C. T. Chan, and C. M. Soukoulis, in Photonic Band Gap Materials, edited by C. M. Soukoulis (Kluwer, Dordrecht, 1996).

[16] We use the band structures like Fig. 1 , where $+\kappa(-\kappa)$ relates to a right-going (left-going) Bloch wave in the pass bands, and corresponds to a right-decaying (left-decaying) wave in the band gaps. When a stationary harmonic plane sound source is put inside the phononic crystal with absorbing boundaries at both ends, a wave with $+\kappa(-\kappa)$ can be generated on the rightand left-hand side of the source.

[17] In the derivation, we use $\int_{-\infty}^{+\infty} e^{i \kappa_{\omega}|p|+i B p} d p=i\left\{\left[1 /\left(\kappa_{\omega}-B\right)\right]\right.$ $\left.+\left[1 /\left(\kappa_{\omega}+B\right)\right]\right\}$ by supposing a very small $\operatorname{Im}(\kappa)$ in pass bands.

[18] X. Hu et al., Phys. Rev. E 71, 055601(R) (2005); J. G. Tolan and J. B. Schneider, J. Acoust. Soc. Am. 114, 2575 (2003); A. Taflove, The Finite-Difference Time-Domain Method (Boston, Artech House, 1998).

[19] We used a miniature MP3 player (cross-sectional area $\approx 0.15 S_{2}$ ) as the sound source and generate the singlefrequency sound file with a software Cool edit Pro (CEP). CEP can also be used to extract the sound pressure data from the recording sound file. 